\title{
The expression of niacin receptor GPR109A on monocytes with age at onset in schizophrenia patients
}

\author{
T.Y. Lin 1 , J.J. Lin ${ }^{2}$, H.H. Tseng 3 , P.S. Chen ${ }^{3}$, S.H. Lin ${ }^{1}$. \\ ${ }^{1}$ Institute of Clinical Medicine, College of Medicine, National Cheng Kung University, Tainan, Taiwan R.O.C. \\ ${ }^{2}$ Department of Psychiatry, Chi Mei Medical Center, Tainan, Taiwan R.O.C.
}

${ }^{3}$ Department of Psychiatry, National Cheng Kung University Hospital, College of Medicine, National Cheng Kung University, Tainan, Taiwan R.O.C.

\section{Objectives}

The goal of this study was to evaluate whether the expression of GPR109A on monocytes might serve as clinically useful biomarker in different age-onset schizophrenia patients.

\section{Background}

The bluntness in niacin skin flush test might reflect abnormalities in membrane fatty acid composition, which could be mediated by a G-protein-coupled receptor called hydroxyl-carboxylic acid receptor 2 (GPR109A) in schizophrenia. The onset age of schizophrenia is related to the severity of the subsequent symptoms, especially the early-onset schizophrenia (EOS). The GPR109A expression levels on peripheral blood in different age-onset

schizophrenia patients remain unknown.

We aimed to determine whether the expression of GPR109A might serve as potential biomarker in different age-onset schizophrenia patients.

\section{Research Design and Methods \\ Participants}

28 patients with schizophrenia and 32 nonpsychotic controls. All patients fulfilled the diagnostic DSM-5 criteria for schizophrenia. The schizophrenia patients that divided into two subgroups by onset age: EOS patients whose onset occurred before the age of 20 years and AOS patients whose onset occurred at or after the age of 20 years.

\section{GPR109A expression}

$8 \mathrm{ml}$ of blood was drawn from each subject by venipuncture into a vacuum tube containing EDTA. Whole blood samples were processed with RosetteSep Human Monocyte Enrichment Cocktail according to the manufacturer's instructions. Buffy coat containing enriched monocytes was isolated and washed twice and with PBS $+2 \%$ FBS. We analyzed the expression of GPR109A on human CD14+ monocytes by FACSCalibur flow cytometer and FlowJO Software.

\section{Data Analyses}

We examined the expression of GPR109A on CD14+ monocytes in different age-onset patients and healthy controls by firth logistic regression.

\begin{tabular}{|c|c|c|c|c|c|c|}
\hline \multirow{4}{*}{ Variables } & \multicolumn{4}{|c|}{ Schizophrenia Patients } & \multicolumn{2}{|c|}{ Healthy Controls } \\
\hline & \multirow{2}{*}{\multicolumn{2}{|c|}{$\begin{array}{l}\text { EOS } \\
(\mathrm{N}=8)\end{array}$}} & \multirow{2}{*}{\multicolumn{2}{|c|}{$\begin{array}{c}\text { AOS } \\
(\mathrm{N}=20)\end{array}$}} & \multirow{2}{*}{\multicolumn{2}{|c|}{$(\mathrm{N}=32)$}} \\
\hline & & & & & & \\
\hline & $\mathrm{N}$ & $\%$ & $\mathrm{~N}$ & $\%$ & $\mathrm{~N}$ & $\%$ \\
\hline Male & 5 & 62.5 & 8 & 40 & 10 & 31.2 \\
\hline Cigarette smoking & 1 & 12.5 & 2 & 10 & 3 & 9.38 \\
\hline Coffee drinking & 2 & 25 & 2 & 10 & 11 & 34.3 \\
\hline History of allergy & 0 & 0 & 0 & 0 & 2 & 6.25 \\
\hline & Mean & SD & Mean & SD & Mean & SD \\
\hline Age (yrs) & 39 & 7 & 51 & 8.7 & 38.8 & 11.6 \\
\hline Onset age (yrs) & 17.7 & 1.2 & 27.8 & 6.2 & - & - \\
\hline BMI & 27.4 & 2.1 & 26.9 & 4.9 & 23.3 & 3.4 \\
\hline Duration (yrs) & 21.5 & 6.9 & 22.8 & 7.9 & - & - \\
\hline Education (yrs) & 12.4 & 2.8 & 11.3 & 4.4 & 16.1 & 4.4 \\
\hline PANSS total & 69.7 & 16.4 & 66.8 & 9.0 & - & - \\
\hline PANSS positive & 16.7 & 1.2 & 15.1 & 4.8 & - & - \\
\hline PANSS negative & 22.3 & 11.8 & 18.1 & 5.5 & - & - \\
\hline PANSS general & 30.1 & 5.6 & 32.7 & 7.7 & - & - \\
\hline
\end{tabular}

EOS, early-onset of schizophrenia; AOS, adult -onset of schizophrenia; PANSS, Positive and Negative Syndrome Scale.

Table 2. Odds ratio in schizophrenia subtypes and healthy controls of GPR109A expression on CD14+ monocytes.

\begin{tabular}{cccc}
\hline & OR in EOS vs AOS & OR in EOS vs HC & OR in AOS vs HC \\
\hline $\begin{array}{c}\text { GPR109A } \\
\text { expression }\end{array}$ & 3.02 & 5.92 & 1.61 \\
\hline
\end{tabular}

EOS, early-onset of schizophrenia; AOS, adult -onset of schizophrenia; HC, healthy controls; OR, odds ratio.

A
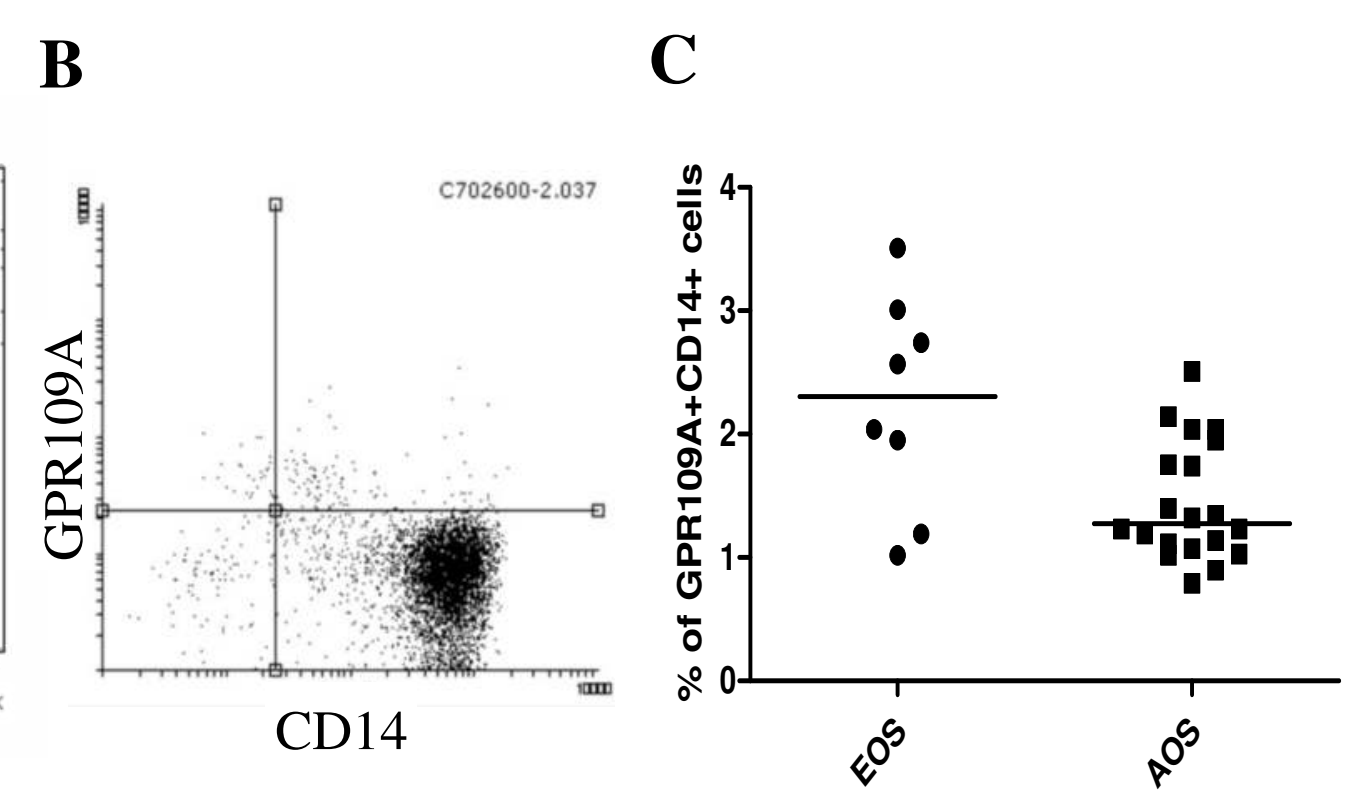

Fig 1. Expression of GPR109A receptor by CD14+ monocytes. FFS/SSC plot for human peripheral monocytes (A). Plot analysis of CD14+GPR109A monocytes (B). GPR109A receptor expression on CD14+ monocytes with EOS and AOS (C).

\section{Conclusion}

The findings provide further insights into GPR109A expression levels as potential biomarker for EOS. However, the small sample size may reduce the power of the study to find significant association with GPR109A and different ageonset schizophrenia patients. 